\title{
Development and Evaluation of Serum CSTI Detection for Early Diagnosis of Esophageal Squamous Cell Carcinoma
}

\author{
Jianwei Wang ${ }^{1,2, *}$ \\ Lili $\mathrm{Yu}^{1,3, *}$ \\ Yulong Sun ${ }^{4, *}$ \\ Liangming Zhang ${ }^{1,3}$ \\ Mingshu Tu ${ }^{1,3}$ \\ Liqing Cail ${ }^{1,3}$ \\ Xiaoqing Yin $^{3,5}$ \\ Xiaojie $\operatorname{Pan}^{1,6}$ \\ Tao Wang (iD 4 \\ Yi Huang (ID) 1,7,8 \\ 'Provincial Clinical College, Fujian \\ Medical University, Fuzhou, 35000I, \\ People's Republic of China; ${ }^{2}$ Department \\ of Clinical Laboratory, Fujian Provincial \\ Hospital South Branch, Fuzhou, 350008, \\ People's Republic of China; ${ }^{3}$ Department \\ of Clinical Laboratory, Fujian Provincial \\ Hospital, Fuzhou, 35000I, People's \\ Republic of China; ${ }^{4}$ Shanghai Liangrun \\ Biomedicine Technology Limited \\ Company, Shanghai, 200000, People's \\ Republic of China; ${ }^{5}$ Integrated Chinese \\ and Western Medicine College, Fujian \\ University of Traditional Chinese \\ Medicine, Fuzhou, 35000I, People's \\ Republic of China; 'Department of \\ Thoracic Surgery, Fujian Provincial \\ Hospital, Fuzhou, 35000I, People's \\ Republic of China; ${ }^{7}$ Central Laboratory, \\ Fujian Provincial Hospital, Fuzhou, \\ 35000I, People's Republic of China; \\ ${ }^{8}$ Center for Experimental Research in \\ Clinical Medicine, Fujian Provincial \\ Hospital, Fuzhou, 35000I, People's \\ Republic of China
}

*These authors contributed equally to this work

Correspondence: Yi Huang; Tao Wang Email hyi8070@I26.com; wangtao@microdiag.com
Purpose: Our pilot study has shown that cystatin SN (CST1) protein is highly expressed in esophageal squamous cell carcinoma (ESCC) tissues. We intend to develop a chemiluminescent enzyme immunoassay (CLEIA) available for serum CST1 detection and define the diagnostic value of CST1 detection for early ESCC patients, and establish a panel of CST1 with traditional tumor markers to improve the diagnostic sensitivity for early ESCC.

Methods: Detection performance of CLEIA for CST1 was evaluated by linearity, detection limit, accuracy, precision, anti-interference and stability. Diagnostic performance of CST1 for early ESCC was evaluated by detecting CST1 of 112 early ESCC, 107 esophageal benign lesions (EBL), and 151 healthy controls (HC). CEA, CYFRA21-1 and SCC-Ag were detected by chemiluminescence immunoassay (CLIA).

Results: The linear range and detection limit of CLEIA for CST1 were $6.25-400 \mathrm{pg} / \mathrm{mL}$ and $1.35 \mathrm{pg} / \mathrm{mL}$, respectively; the average recovery rate was $102.65 \%$; CVs of intra-batch precision and inter-batch precision were $<1 / 4 \mathrm{TEa}$ and $<1 / 3 \mathrm{TEa}$, respectively; 8 interferents including 7 common interferents and CST4 had no interference on CST1 detection; stability evaluation showed good sample and reagent stability. The level and positive rate of CST1 in early ESCC group were significantly higher than those in EBL/HC groups $(P<0.05)$. The diagnostic sensitivity of CST1 for early ESCC was $31.25 \%$ (specificity $92.64 \%$, AUC 0.654 ). The diagnostic sensitivity of traditional tumor markers ranged from $16.07 \%$ to $28.57 \%$, at $>93.0 \%$ specificity, and SCC-Ag showed the highest AUC (0.709). Combination of CST1 and CEA, SCC-Ag exhibited the highest AUC up to 0.736 (sensitivity $49.11 \%$, specificity $89.53 \%$ ).

Conclusion: CLEIA has excellent detection performance for CST1. CST1 might be a prospective serological biomarker for early diagnosis of ESCC, while combination of CST1 and CEA, SCC-Ag might improve the early diagnostic performance.

Keywords: esophageal squamous cell carcinoma, cystatin SN, chemiluminescence enzyme immunoassay, methodological evaluation, early diagnosis

\section{Introduction}

Esophageal cancer is one of the most common malignant tumors in the clinic. It originates from the esophageal epithelium and manifests as a series of symptoms, such as swallowing pain, dysphagia, and weight loss. ${ }^{1}$ Esophageal cancer is mainly divided into esophageal adenocarcinoma (EAC) and esophageal squamous cell carcinoma (ESCC). In recent years, the incidence of ESCC is significantly higher than that of EAC in $>90 \%$ of countries, of which China has the highest incidence of 
ESCC in the world, ${ }^{2}$ with 572,034 new cases reported in 2018. ${ }^{3}$ As a highly invasive tumor, ESCC development often leads to lymph node metastasis, showing highly malignant biological behavior. More than $80 \%$ of patients have been at the middle and advanced stage when they visit the doctor, with a dismal 5-year survival rate of $15 \%$ to $20 \%{ }^{4,5}$ Therefore, early diagnosis and timely treatment are critical for improving the prognosis and survival rate of ESCC patients. Unfortunately, fiber endoscopy and mucosal biopsy are demonstrated to be not available for routine screening for the patients with ESCC at early stage due to several shortages such as relatively complex operation and uncomfortable invasion experience, etc. ${ }^{6}$ Serological biomarkers have the advantages of easy accessibility, convenient detection, and high acceptance by patients and play a critical role in the early screening of ESCC. However, the traditional tumor markers, CEA, CYFRA21-1 and SCC-Ag, present a different degree of detection sensitivity or specificity problems, which limit their clinical value for the early diagnosis of ESCC. ${ }^{7,8}$ Therefore, finding new prospective serological markers is very essential for the patients with ESCC at early stage.

Cystatin (CST) was first isolated from egg white by Anastasi et al using affinity chromatography, with the feature of inhibitory effect on cysteine protease. ${ }^{9}$ CST1 gene is located on chromosome 20p11.21 and encodes cystatin SN protein that belongs to the second subfamily of cystatin superfamily, which includes 7 exocrine proteins: Cystatin SN (CST1), Cystatin SA (CST2), Cystatin C (CST3), Cystatin A (CST4), Cystatin D (CST5), Cystatin E/M (CST6), and Cystatin F (CST7). Other than CST3, which is widely expressed in various body fluids and tissues, the other 6 members in the second subfamily of cystatin superfamily present specific distribution limitations in different body fluids and tissues. For example, CST1 is found to be limitedly expressed in seminal plasma, lacrimal fluid, cystic fluid, submandibular gland, lacrimal gland, and gallbladder. ${ }^{10-12}$ A balance between CST and cysteine protease must be maintained in organisms because its breakdown is speculated to lead to malignancies. ${ }^{13}$ Recently, some CSTs have been reported to be closely correlated with malignancies, ${ }^{14}$ of which CST1 was shown high expression in cancerous tissues of some malignancies, including colorectal cancer, gastric cancer, liver cancer, pancreatic cancer, and breast cancer, and then promoted cancerous cell proliferation, migration, and invasion. ${ }^{15-19}$ Additionally, CST1 is found to be a downstream effector of transcription factor $\mathrm{TCF}^{20}$ involved in the occurrence and development of gastric cancer via $\mathrm{Wnt} / \beta$-catenin/TCF signal transmission; ${ }^{16}$ in liver cancer, CST1 promoted the epithelial-mesenchymal transition (EMT) process of cancerous cells by regulating the PI3K/Akt signaling pathway. ${ }^{17}$

Considered that the correlation between CST1 and ESCC has not yet been clarified up to now, it is worth exploring whether there is ectopic high expression of CST1 in both cancerous tissues and sera of ESCC patients. As expected, the result of immunohistochemical (IHC) staining showed CST1 protein was aberrantly expressed in $81.8 \%$ of cancerous tissues while negative in all matched paracancerous tissues of 22 early ESCC patients in our pilot study. Therefore, it is reasonable to speculate that aberrant expression of CST1 might also exist in sera of the patients with early ESCC. In this study, we intend to develop a chemiluminescent enzyme immunoassay (CLEIA) available for serum CST1 detection and define the diagnostic value of serum CST1 detection for early ESCC patients, and then determine an optimal panel of serum CST1 with traditional tumor markers to further improve the diagnostic performance for ESCC patients at early stage.

\section{Materials and Methods Study Participants}

Three-hundred and seventy serum samples from Fujian Provincial Hospital during the period from January 2017 to December 2019 were derived from 112 patients with early esophageal squamous cell carcinoma (ESCC), 107 patients with esophageal benign lesions (EBL) and 151 healthy controls (HC). All early ESCC patients at TNM 0/I/II stage were confirmed by endoscopy combined with cytology or histopathology and did not receive any anti-tumor therapy such as surgery, chemoradiotherapy, and molecular targeted therapy. The EBL group included esophageal benign tumors, benign ulcer of esophagus, esophageal erosions and reflux esophagitis, all of whom were inpatients and outpatients from Fujian Provincial Hospital during the same period. The HC group included 151 healthy adults receiving the physical examination at the Physical Examination Center in Fujian Provincial Hospital and showed no evidence of malignancies, EBL, etc. The clinical and pathological data are listed in Table $1.5 \mathrm{~mL}$ peripheral blood from each subject was collected and the serum was separated at $3000 \mathrm{rpm}$ 
Table I Clinical Characteristics of the Early ESCC, EBL and HC Groups

\begin{tabular}{|c|c|c|c|c|c|c|c|c|c|c|}
\hline \multirow[t]{2}{*}{ Variable } & \multicolumn{3}{|c|}{ Early ESCC $(n=1 \mid 2)$} & \multicolumn{3}{|c|}{ EBL $(n=107)$} & \multicolumn{3}{|c|}{$H C(n=|5|)$} & \multirow[t]{2}{*}{$P$} \\
\hline & No. & Mean & $\%$ & No. & Mean & $\%$ & No. & Mean & $\%$ & \\
\hline Age (years): & & & & & & & & & & $>0.05$ \\
\hline Mean & & 60.5 & & & 60.0 & & & 58.3 & & \\
\hline Standard deviation & & 9.5 & & & 11.1 & & & 8.6 & & \\
\hline Sex: & & & & & & & & & & $>0.05$ \\
\hline Male & 78 & & 69.6 & 67 & & 62.6 & 102 & & 67.5 & \\
\hline Female & 34 & & 30.4 & 40 & & 37.4 & 49 & & 32.5 & \\
\hline Smoking history: & 35 & & 31.3 & 32 & & 29.9 & 43 & & 28.5 & $>0.05$ \\
\hline Drinking history: & 20 & & 17.9 & 14 & & 13.1 & 19 & & 12.6 & $>0.05$ \\
\hline Stage: & & & & & & & & & & \\
\hline 0 & 33 & & 29.5 & & & & & & & \\
\hline I & 31 & & 27.7 & & & & & & & \\
\hline II & 48 & & 42.8 & & & & & & & \\
\hline EBL: & & & & & & & & & & \\
\hline Esophageal benign tumors & & & & 54 & & 50.5 & & & & \\
\hline Benign ulcer of esophagus & & & & 8 & & 7.5 & & & & \\
\hline Esophageal erosions & & & & 32 & & 29.9 & & & & \\
\hline Reflux esophagitis & & & & 13 & & 12.1 & & & & \\
\hline
\end{tabular}

for $5 \mathrm{~min}$ and stored at $-80^{\circ} \mathrm{C}$ before use. Twenty-two pairs of ESCC tissues and matched paracancerous tissues for IHC staining were randomly selected from 112 cases with ESCC at early stage. The Institutional Review Board of Fujian Provincial Hospital approved this study in accordance with the principles of the Declaration of Helsinki, and all participants provided written informed consent.

\section{IHC Staining}

Formalin-fixed, paraffin-embedded, 3-mm thick sections were deparaffinized and rehydrated. IHC SP staining kit (Fuzhou Maixin Biotechnology Limited Company, China) was used for staining according to the manufacturer's instructions. The sections were, respectively, incubated with primary antibody against CST1 (1:100, Abcam (Shanghai) Trading Limited Company, China) overnight at $4{ }^{\circ} \mathrm{C}$, followed by incubation with the biotinylated secondary antibody for 10 min at room temperature, and horseradish peroxidaseconjugated streptavidin for $10 \mathrm{~min}$ at room temperature. The immunoreactivities were visualized brown with diaminobenzidine (DAB kit; Lab Vision) and counterstained with Mayer's hematoxylin. ESCC tissue sections always positive after multiple tests and verification were used as the positive control, and PBS was used as the negative control instead of the primary antibody, keeping all other steps in the process the same. The intensity of staining was graded as: $0=$ undetectable, $1+=$ weak staining, $2+=$ moderate staining, and $3+=$ strong staining.

\section{Development of Chemiluminescent Enzyme Immunoassay (CLEIA) for Serum CSTI Detection}

$1.6 \mu \mathrm{g} / \mathrm{mL}$ mouse monoclonal antibody against CST1 (Shanghai Liangrun Biomedicine Technology Limited Company, China) diluted with CBS buffer was coated onto 96-well plates, $100 \mu \mathrm{L} /$ well, at $4{ }^{\circ} \mathrm{C}$ overnight. After 1 time wash with PBST, $200 \mu \mathrm{L}$ microplate stabilizer I (Huzhou Yingchuang Biotechnology Limited Company, China) was added and incubated at $37{ }^{\circ} \mathrm{C}$ for $60 \mathrm{~min}$. After 3 times wash with PBST, 100 $\mu \mathrm{L}$ CST1 calibrator (CST1 recombinant antigen, Shanghai Liangrun Biomedicine Technology Limited Company, China) or serum sample was added and 
incubated at $37^{\circ} \mathrm{C}$ for $60 \mathrm{~min}$. After 5 times wash with PBST, $100 \mu \mathrm{L}$ horseradish peroxidase (HRP) labeled rat monoclonal antibody against CST1 (1:2000 dilution, Shanghai Liangrun Biomedicine Technology Limited Company, China) was added and incubated at $37{ }^{\circ} \mathrm{C}$ for $30 \mathrm{~min}$. After 5 times wash with PBST, 100 $\mu \mathrm{L}$ luminescent solution (substrate A containing luminescent agent and enhancer mixed with substrate $\mathrm{B}$ containing hydrogen peroxide at a ratio of 1:1 before use) was added and incubated at room temperature for 3 min. RLU was measured on Antu bio LUMO chemiluminescence analyzer (Shanghai Antu Biotechnology Limited Company, China), and the CST1 level was calculated according to the standard curve.

\section{Evaluation of CLEIA for Serum CSTI Detection}

The detection limit and linearity the calibrator $(0 \mathrm{pg} / \mathrm{mL})$ with no analyte was repeatedly detected by CLEIA for 20 times, and the mean $(\bar{X})$ value of RLU and standard deviation (SD) were calculated. $\bar{X}+3 \mathrm{SD}$ was equal to the detection limit. After serial double dilution, each level of the CST1 calibrator (CST1 recombinant antigen, Shanghai Liangrun Biomedicine Technology Limited Company, China) was detected twice, and the average value of RLU was calculated. The linear regression curve was drawn by using $\log (\mathrm{C})$ as the ordinate $\mathrm{Y}$ and $\log$ (RLU) as the abscissa $X$. The regression equation was " $\log (C)=a * \log (R L U)+b "$. The $a$ and $b$ in the formula were the parameters of CLEIA, which determined the conversion relationship between the RLU value and the CST1 level of the sample.

Accuracy $900 \mu \mathrm{L}$ basic serum sample added to $100 \mu \mathrm{L}$ distilled water was taken as the control sample; $900 \mu \mathrm{L}$ basic serum sample added to $8 \mu \mathrm{L}$ calibrator of $200 \mathrm{pg} / \mathrm{mL}$ CST1 and $92 \mu \mathrm{L}$ distilled water was taken as the recovery sample 1 ; $900 \mu \mathrm{L}$ basic serum sample added to $48 \mu \mathrm{L}$ calibrator of 200 $\mathrm{pg} / \mathrm{mL} \mathrm{CST1}$ and $52 \mu \mathrm{L}$ distilled water was taken as recovery sample 2; and $900 \mu \mathrm{L}$ basic serum sample added to $72 \mu \mathrm{L}$ calibrator of $200 \mathrm{pg} / \mathrm{mL} \mathrm{CST} 1$ and $28 \mu \mathrm{L}$ distilled water was taken as recovery sample 3 . Each sample was detected twice and the mean level was calculated. The recovery rate was calculated according to the following formula:

$$
R=\frac{\left(V_{S}+V_{0}\right) X\left(C_{1}-C_{0}\right)}{V_{S} C_{S}}
$$

In the formula, the final detected level was $\mathrm{C}_{1}$, and the basic serum sample level and volume were $C_{0}$ and $V_{0}$, respectively. The calibrator level and volume were $C_{S}$ and $\mathrm{V}_{\mathrm{S}}$, respectively. The recovery rate was $\mathrm{R}$. $\mathrm{R}$ within the range of $85 \%$ to $115 \%$ was considered to be acceptable.

Precision Precision evaluation was performed according to CLSI ep15-a2 standard: Low and high CST1 levels of samples were detected for 5 days, respectively, each level of which was repeatedly detected 3 times and then the average value was calculated.

Anti-interference Interference evaluation was performed according to WS-T 416-2013 interference experiment guide: Seven common interferents, that is, hemoglobin $(\mathrm{Hb})$ of $2 \mathrm{~g} / \mathrm{L}$, rheumatoid factor (RF) of $750 \mathrm{IU} / \mathrm{mL}$, bilirubin (Bil) of $342 \mu \mathrm{mol} / \mathrm{L}$, triglyceride (TG) of $37 \mathrm{mmol} / \mathrm{L}$, alpha fetoprotein (AFP) of $5 \mathrm{mg} / \mathrm{dL}$, carcinoembryonic antigen (CEA) of $0.2 \mu \mathrm{g} / \mathrm{mL}$ and vita$\min \mathrm{C}$ (VitC) of $12.3 \mathrm{mg} / \mathrm{dL}$, and CST4 (CST4 recombinant protein, Shanghai Liangrun Biomedicine Technology Limited Company, China) of $800 \mathrm{pg} / \mathrm{mL}$, were added to the basic serum samples with CST1 levels of $20.95 \mathrm{pg} / \mathrm{mL}$ and $65.39 \mathrm{pg} / \mathrm{mL}$ at a ratio of $1: 9$, respectively. Each sample was detected 3 times, and the relative deviation ( $\delta)<10 \%$ was considered to be acceptable.

Sample stability Low levels $(23.74 \mathrm{pg} / \mathrm{mL})$, medium levels $(81.60 \mathrm{pg} / \mathrm{mL})$ and high levels $(197.03 \mathrm{pg} / \mathrm{mL})$ of serum samples stored at $4{ }^{\circ} \mathrm{C}$ and $25^{\circ} \mathrm{C}$ for $0,1,2,3,4,5$, 6 and $7 \mathrm{~d}$ were detected, respectively. Each sample was detected twice, and $\delta$ was calculated by comparing the results of different time points with that for 0 d. $\delta<10 \%$ was considered to be acceptable.

Reagent stability The reagents stored at $25^{\circ} \mathrm{C}$ for 0,1 , 3,5 and $7 \mathrm{~d}$ were used to detect CST1 calibrators and serum samples, respectively. The reagents stored at $37{ }^{\circ} \mathrm{C}$ for $0,1,3,5$ and $7 \mathrm{~d}$ were used to detect CST1 calibrators and serum samples, respectively. The reagents stored at 4 ${ }^{\circ} \mathrm{C}$ for $0,1,3$ and $6 \mathrm{~m}$ were used to detect CST1 calibrators and serum samples, respectively. Each sample was detected twice, and $\delta<10 \%$ was considered to be acceptable.

\section{Evaluation of Diagnostic Performance of Serum CSTI for Early ESCC Patients by CLEIA}

The levels of serum CST1 of 112 cases in early ESCC group, 107 cases in EBL group and 151 cases in HC group were detected by CLEIA. The difference of serum CST1 level between the early ESCC group and control group (EBL+HC group) was evaluated by the Mann-Whitney 
$U$-test. The 95th percentile of serum CST1 level in HC group was used to determine the cutoff value for the diagnosis of early ESCC.

\section{Detection of Three Traditional Tumor Markers, CEA, CYFRA2I-I and SCC-Ag, for Early ESCC Patients}

The levels of serum CEA and CYFRA21-1, and SCC-Ag of 112 cases in early ESCC group, 107 cases in EBL group and 151 cases in HC group were detected by chemiluminescence immunoassay (CLIA) in cobas 602 analyser (Roche, Mannheim, Germany), and Architect i2000SR analyser (Abbott Laboratories, Abbott Park, IL, USA), respectively. All procedures were strictly carried out according to the manufacturer's instruction. The cutoff values of serum CEA, CYFRA21-1 and SCC-Ag were $5.0 \mathrm{ng} / \mathrm{mL}, 3.3 \mathrm{ng} / \mathrm{mL}$ and $1.5 \mathrm{ng} / \mathrm{mL}$, respectively.

\section{Statistical Analysis}

SPSS 25.0 statistical software was used for the analysis of the experimental data. GraphPad Prism 5 was used to draw charts. SPSS 25.0 statistical software was used to draw ROC curves and AUC value was calculated. $P<0.05$ was considered a significant difference.

\section{Results}

\section{Expression of CSTI Protein in ESCC} Tissues

The results of IHC staining showed that CST1 protein was localized in the cytoplasm and focally or diffusely distributed brown granules in ESCC tissues (Figure 1A), while it was negative in matched paracancerous tissue (Figure 1B). The positive expression rate of CST1 protein in ESCC tissues was $81.8 \%(18 / 22)$, which was significantly higher than that in matched paracancerous tissue $(0.00 \%, 0 / 22$; $P<0.01)$.

\section{Evaluation of the Detection Performance of CLEIA}

Detection limit and linearity The $\bar{X}$ and SD obtained from 20 parallel detections of the calibrator $(0 \mathrm{pg} / \mathrm{mL})$ were 8117 and 157.7, respectively, and the detection limit $(\bar{X}+3 \mathrm{SD})$ was determined to be $1.35 \mathrm{pg} / \mathrm{mL}$ (Table 2). Eight levels of CST1 calibrator by double dilution within the range of $3.125-400 \mathrm{pg} / \mathrm{mL}$ were detected twice, and then the linear regression curve was plotted (Figure 2). However, as the level of CST1 calibrator was $<6.25 \mathrm{pg} /$ $\mathrm{mL}$, the linear relationship was not significant (data not shown). So, $6.25-400 \mathrm{pg} / \mathrm{mL}$ was selected as the linear range of quantitative detection of serum CST1 of CLEIA. $\log (\mathrm{C})$ and $\log (\mathrm{RLU})$ of the 7 concentration points were calculated and then the linear regression equation was deduced as follows:

$\log (\mathrm{C})=1.213 \log (\mathrm{RLU})-4.079\left(\mathrm{R}^{2}=0.9935, \mathrm{P}<0.01, \mathrm{~N}=7\right)$

Accuracy The detection results of recovery experiment showed that the average recovery rate was calculated to be $102.65 \%$, within the range of $85-115 \%$ (Table 3 ).

Precision The CVs of intra-batch of low and high level were $4.43 \%$ and $1.94 \%$, respectively, which were $<1 / 4 \mathrm{TEa}$ (according to CLIA'88, 25\%). The CVs of inter-batch of low and high level were $1.39 \%, 1.90 \%$, respectively, which were $<1 / 3 \mathrm{TEa}$ (according to CLIA'88, 25\%) (Table 4).

Anti-interference evaluation The results of antiinterference ability evaluation of CLEIA showed that eight interferents, that is, seven common interferents and
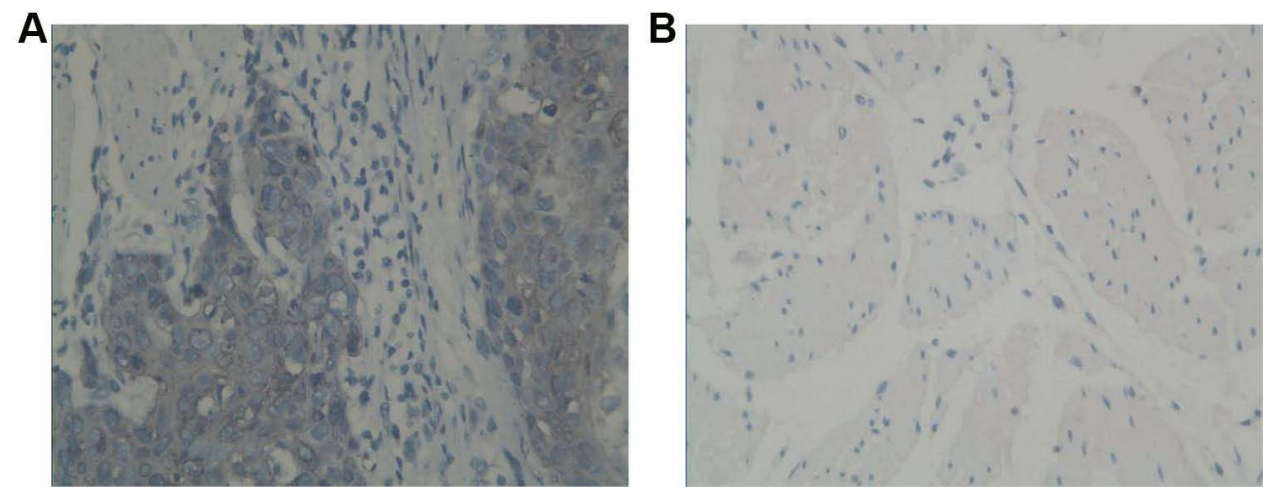

Figure I IHC staining of CSTI in ESCC tissue and paracancerous tissue ( $\times 400)$. (A) Strong expression of CSTI with $3+$ staining in ESCC tissue. (B) Negative expression of CSTI in paracancerous tissue. 
Table 2 Detection Limit of CLEIA for CSTI

\begin{tabular}{|l|l|l|l|}
\hline & RLU & & RLU \\
\hline Measured value 1 & 8124 & Measured value II & 7986 \\
Measured value 2 & 7913 & Measured value 12 & 8278 \\
Measured value 3 & 7873 & Measured value 13 & 8501 \\
Measured value 4 & 8032 & Measured value 14 & 8224 \\
Measured value 5 & 8206 & Measured value 15 & 8146 \\
Measured value 6 & 8169 & Measured value 16 & 8004 \\
Measured value 7 & 8043 & Measured value 17 & 8206 \\
Measured value 8 & 7884 & Measured value 18 & 8004 \\
Measured value 9 & 8263 & Measured value 19 & 8231 \\
Measured value 10 & 8237 & Measured value 20 & 8023 \\
\hline \multicolumn{4}{|c|}{$\bar{X}=81$ I7 } \\
\hline
\end{tabular}

CST4, had no interference on CLEIA for serum CST1 detection (Table 5).

Sample stability The detection results of the low, medium and high levels of samples stored at $4{ }^{\circ} \mathrm{C}$ for $0,1,2,3,4,5,6$ and $7 \mathrm{~d}$ are shown in Figure 3A, all of which $\delta$ calculated by comparing the results for different time points with that for 0 $\mathrm{d}$ were $<10 \%$. The detection results of the low, medium and high levels of samples stored at $25^{\circ} \mathrm{C}$ for $0,1,2,3,4,5,6$ and $7 \mathrm{~d}$ are shown in Figure 3B, of which $\delta$ for 2, 3, 4, 5, 6 and $7 \mathrm{~d}$ were $>10 \%$, illustrating that storage at $25^{\circ} \mathrm{C}$ for more than $2 \mathrm{~d}$ had a certain impact on the sample stability.

Reagent stability Thermal stability: The detection results of the reagent stored at $25^{\circ} \mathrm{C}$ and $37^{\circ} \mathrm{C}$ for 0,1 , 3, 5, and $7 \mathrm{~d}$ are shown in Figure 4, all of which $\delta$ calculated by comparing the results for different time

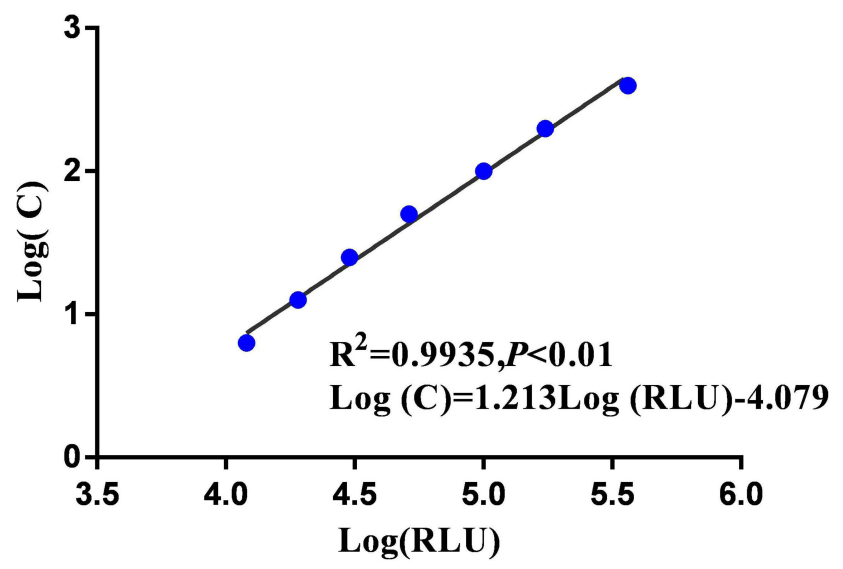

Figure 2 Standard curve for CLEIA. The RLU value is the mean of each diluted level detected twice; $\mathrm{C}$ is the diluted level $(\mathrm{pg} / \mathrm{mL})$ of CSTI calibrator. The same base logarithms of RLU and $C$ are taken as $X$ and $Y$ axis, respectively, to draw the curve. points with that for $0 \mathrm{~d}$ were $<10 \%$. Validity period stability: The detection results of the reagent stored at $4{ }^{\circ} \mathrm{C}$ for $0,1,3$ and $6 \mathrm{~m}$ are shown in Figure 5, all of which $\delta$ calculated by comparing the results for different time points with that for $0 \mathrm{~m}$ were $<10 \%$.

\section{Evaluation of Diagnostic Performance of Serum CSTI for Early ESCC Patients by CLEIA}

The detection results of the serum samples by CLEIA are presented in Figure 6A. The level of serum CST1 in early ESCC group was significantly higher than that in the control group (EBL+HC group) $(P<0.05)$, while there was no significant difference between EBL group and HC group $(P>0.05)$. The 95 th percentile of serum CST1 level in $151 \mathrm{HC}$ was $68.33 \mathrm{pg} / \mathrm{mL}$; as the cutoff level was set at 68.33 , sensitivity and specificity of serum CST1 for early ESCC patients were $31.25 \%$ (35/112) and $92.64 \%$ (239/258), respectively. The sensitivity of CST1 in early ESCC group was significantly higher than that in EBL group $(6.54 \%, 7 / 107)$ and $\mathrm{HC}$ group $(7.95 \%, 12 / 151)$ $(P<0.05)$. Analysis of the ROC (early ESCC vs EBL $+\mathrm{HC}$ ) revealed that the AUC of serum CST1 for early ESCC patients was $0.654(P<0.05)$ (Figure 6A).

\section{Diagnostic Performance of Traditional Tumor Markers for Early ESCC Patients}

The level and positive rate of CEA in early ESCC group were significantly higher than those in $\mathrm{EBL} / \mathrm{HC}$ group $(P<0.05)$. The positive rates of CYFRA21-1 and SCC-Ag in early ESCC group were significantly higher than those in EBL/HC group $(P<0.01)$, while there were no significant differences of the levels of CYFRA21-1 and SCC-Ag between early ESCC group and EBL/HC group $(P>0.05)$. The diagnostic sensitivity of three traditional tumor markers for early ESCC patients ranged from 16.07\%-28.57\%, at $>93.0 \%$ specificity, of which SCC-Ag showed the highest AUC, up to 0.709 (Figure 6B-D).

\section{Different Combinations of CSTI and Three Traditional Tumor Markers for Early Diagnosis of ESCC Patients}

Among all possible combinations of CST1 and three traditional tumor markers, a panel of CST1 with CEA and SCC-Ag exhibited the highest AUC up to 0.736 (95\% CI 
Table 3 Accuracy Evaluation of CLEIA for CSTI

\begin{tabular}{|c|c|c|c|c|c|c|c|c|}
\hline \multirow[t]{2}{*}{ CSTI (pg/mL) } & \multicolumn{2}{|c|}{ Sample I } & \multicolumn{2}{|c|}{ Sample 2} & \multicolumn{2}{|c|}{ Sample 3} & \multicolumn{2}{|c|}{ Basic Sample } \\
\hline & $\mathbf{I}$ & 2 & $\mathbf{I}$ & 2 & $\mathbf{I}$ & 2 & $\mathbf{I}$ & 2 \\
\hline Detection level & 27.70 & 26.24 & 33.94 & 34.52 & 41.37 & 40.11 & 24.58 & 25.46 \\
\hline Mean & \multicolumn{2}{|c|}{26.97} & \multicolumn{2}{|c|}{34.23} & \multicolumn{2}{|c|}{40.74} & \multicolumn{2}{|c|}{25.02} \\
\hline Recovery level & \multicolumn{2}{|c|}{1.95} & \multicolumn{2}{|c|}{9.21} & \multicolumn{2}{|c|}{15.72} & & \\
\hline Additive level & \multicolumn{2}{|c|}{1.76} & \multicolumn{2}{|c|}{10.12} & \multicolumn{2}{|c|}{$|4.8|$} & & \\
\hline Recovery rate (\%) & \multicolumn{2}{|c|}{110.80} & \multicolumn{2}{|c|}{91.01} & \multicolumn{2}{|c|}{106.14} & & \\
\hline Mean (\%) & \multicolumn{6}{|c|}{102.65} & & \\
\hline
\end{tabular}

Table 4 Precision Evaluation of CLEIA for CSTI

\begin{tabular}{|l|c|c|c|c|c|c|}
\hline \multirow{2}{*}{ CSTI (pg/mL) } & \multicolumn{3}{|c|}{ Intra-Batch Precision } & \multicolumn{3}{c|}{ Inter-Batch Precision } \\
\cline { 2 - 7 } & SD (pg/mL) & CV (\%) & I/4 TEa (\%) & SD (pg/mL) & CV (\%) & I/3 TEa (\%) \\
\hline 28.25 & 1.252 & 4.43 & 6.25 & 1.291 & 1.39 & 8.33 \\
92.92 & 1.805 & 1.94 & 6.25 & 1.770 & 1.90 & 8.33 \\
\hline
\end{tabular}

Abbreviations: SD, standard deviation; $\mathrm{CV}$, coefficient of variation; $1 / 4 \mathrm{TEa}, \mathrm{I} / 4$ total error allowance; 1/3 TEa, I/3 total error allowance.

Table 5 Evaluation of Anti-Interference Ability of CLEIA for CSTI

\begin{tabular}{|c|c|c|c|c|c|c|}
\hline \multirow[t]{2}{*}{ Interferent } & \multicolumn{3}{|c|}{ Sample I } & \multicolumn{3}{|c|}{ Sample 2} \\
\hline & Basic (pg/mL) & Test (pg/mL) & Bias (\%) & Basic (pg/mL) & Test (pg/mL) & Bias (\%) \\
\hline $\mathrm{Hb}(2 \mathrm{~g} / \mathrm{L})$ & 20.67 & 21.36 & 3.34 & 62.17 & 65.39 & 5.18 \\
\hline RF (750 IU/mL) & 20.67 & 22.28 & 7.79 & 62.17 & 65.65 & 5.60 \\
\hline Bil $(342 \mu \mathrm{mol} / \mathrm{L})$ & 20.67 & 22.63 & 9.48 & 62.17 & 66.04 & 6.22 \\
\hline TG $(37 \mathrm{mmol} / \mathrm{L})$ & 20.67 & 22.52 & 8.95 & 62.17 & 66.14 & 6.39 \\
\hline AFP $(5 \mathrm{mg} / \mathrm{dL})$ & 20.67 & 22.07 & 6.77 & 62.17 & 65.96 & 6.10 \\
\hline CEA $(0.2 \mu \mathrm{g} / \mathrm{mL})$ & 20.67 & 22.34 & 8.08 & 62.17 & 67.54 & 8.64 \\
\hline VitC (I $2.3 \mathrm{mg} / \mathrm{dL})$ & 20.67 & 22.38 & 8.27 & 62.17 & 65.30 & 5.03 \\
\hline CST4 $(800 \mathrm{pg} / \mathrm{mL})$ & 20.67 & 22.13 & 7.06 & 62.17 & 66.89 & 7.59 \\
\hline
\end{tabular}

$0.678-0.793)$, with sensitivity $49.11 \%$ at specificity $89.53 \%$ (Figure 6E, Table 6).

\section{Discussion}

The recent reports on the correlation of CST1 with the diagnosis, illness monitoring, and prognosis evaluation of some malignancies have gained increasing attention. Chen et al found the aberrant expression of CST1 existed in both gastric cancer tissues and cells, and patients with high CST1 expression had poor outcomes. ${ }^{21}$ Dai et al demonstrated that high CST1 expression was negatively correlated with survival of breast cancer patients and might serve as an independent prognostic factor for breast cancer, the same as ER status and nodal status. ${ }^{19}$ Additionally, serum CST1 was also reported to be a valuable diagnostic biomarker for colorectal cancer by enzyme-linked immunosorbent assay (ELISA), manifested as a marked increase in sera of patients with colorectal cancer. ${ }^{15}$ Therefore, although the correlation of CST1 with ESCC has not yet been elucidated up to now, it is expected that the aberrantly high expression of CST1 might exist in cancerous tissues and sera of ESCC patients.

IHC staining was first performed to demonstrate that aberrantly high expression of CST1 protein existed in the ESCC tissues, as evidenced by the positive rate of $81.8 \%$ $(18 / 22)$ in cancerous tissues significantly higher than $0 \%$ 
A

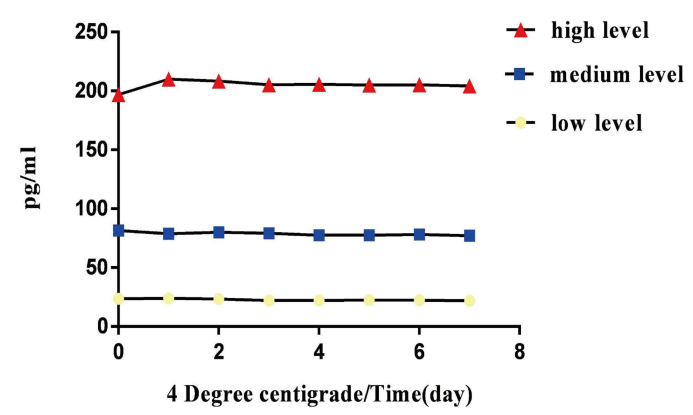

B

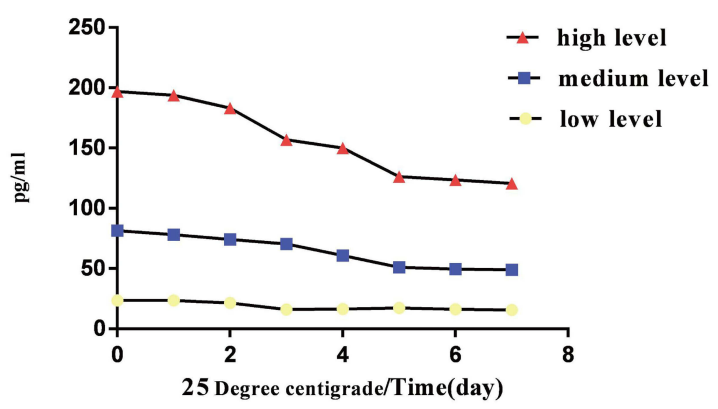

Figure 3 Line chart of quantitative detection results for low, medium and high levels of the serum samples stored at $4{ }^{\circ} \mathrm{C}$ and $25^{\circ} \mathrm{C}$ for eight time points. (A) The quantitative detection results of the low, medium and high levels of the serum samples stored at $4{ }^{\circ} \mathrm{C}$ for $0,1,2,3,4,5,6$ and $7 \mathrm{~d}$. (B) The quantitative detection results of the low, medium and high levels of the serum samples stored at $25^{\circ} \mathrm{C}$ for $0,1,2,3,4,5,6$ and $7 \mathrm{~d}$.

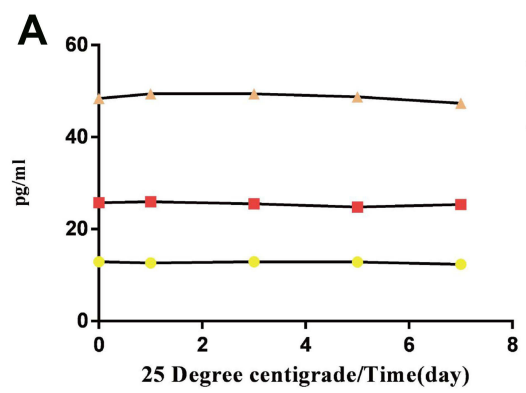

C

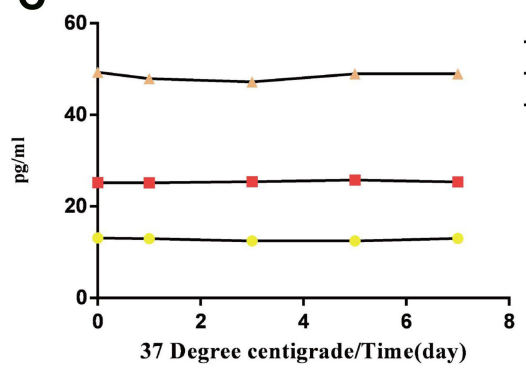

B

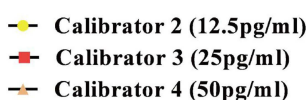

- - Calibrator $4(50 \mathrm{pg} / \mathrm{ml})$

$\overline{\mathrm{g}}$

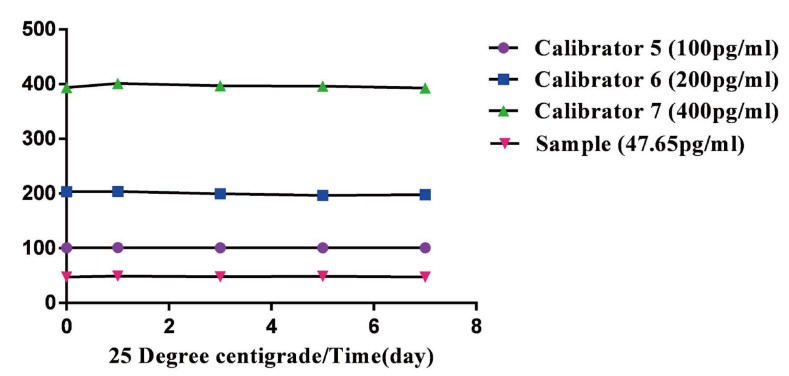

D

-- Calibrator $2(12.5 \mathrm{pg} / \mathrm{ml})$

- Calibrator $3(25 \mathrm{pg} / \mathrm{ml})$

- - Calibrator $4(50 \mathrm{pg} / \mathrm{ml})$

Figure 4 Line chart of quantitative detection results for CSTI calibrators and serum samples by the reagents stored at $25^{\circ} \mathrm{C}$ and $37^{\circ} \mathrm{C}$ for five time points. The quantitative detection results of the reagents stored at $25^{\circ} \mathrm{C}$ for $0,1,3,5$ and $7 \mathrm{~d}$ are presented in $(\mathbf{A})$ and $(\mathbf{B})$. The quantitative detection results of the reagents stored at $37{ }^{\circ} \mathrm{C}$ for 0 , I, 3, 5 and $7 \mathrm{~d}$ are presented in (C) and (D).

$(0 / 22)$ in paracancerous tissues $(\mathrm{P}<0.01)$. Subsequently, we developed a CLEIA available for serum CST1 detection and a systematic methodological evaluation was performed. It was shown that CLEIA has wide linear range and good detection limit of CLEIA for serum CST1, manifested as $6.25-400 \mathrm{pg} / \mathrm{mL}$ and $1.35 \mathrm{pg} / \mathrm{mL}$, respectively; high accuracy with average recovery rate of $102.65 \%$ (85-115\%); good precision with CVs of $4.43 \%$ and $1.94 \%$ within batches for low and high CST1 level, respectively, and CVs of $1.39 \%$ and $1.90 \%$ between batches for low and high CST1 level, respectively; adequate anti-interference ability of eight interferents, that is, seven common interferents and CST4; good $4{ }^{\circ} \mathrm{C}$ stability of sample, and $25^{\circ} \mathrm{C}, 37^{\circ} \mathrm{C}$ thermal stability as well as 4 ${ }^{\circ} \mathrm{C}$ validity period stability of reagent. These findings indicated that the developed CLEIA presents excellent performance for meeting the stable and reliable detection of serum CST1 level of ESCC patients.

In addition, we performed the detection of CST1 in a cohort of 370 serum samples (112 early ESCC 

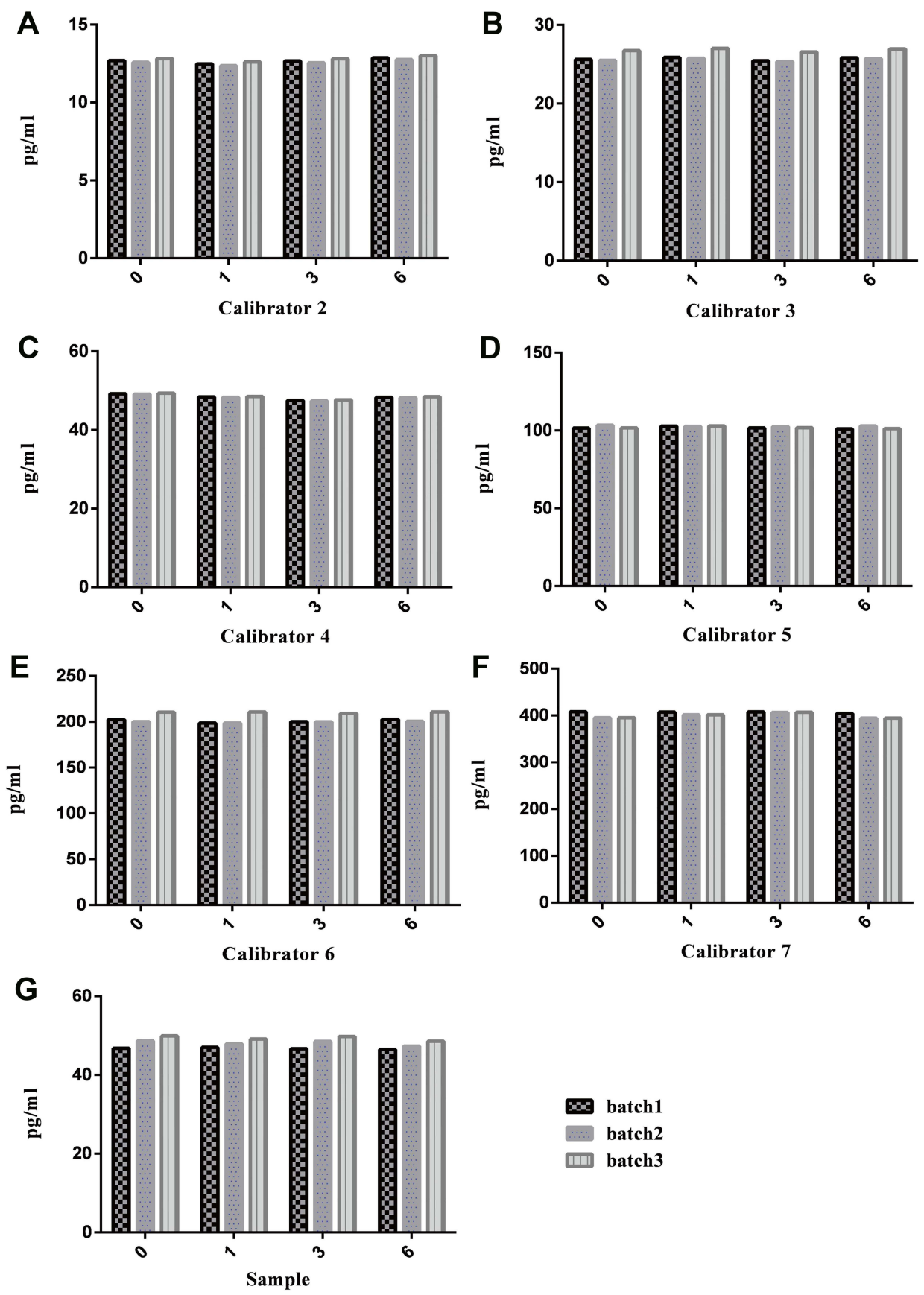

Figure 5 Histogram of quantitative detection results for CSTI by three batches reagents stored at $4{ }^{\circ} \mathrm{C}$ for four time points. The quantitative detection results for CSTI calibrator 2 (I2.5 pg/mL), $3(25 \mathrm{pg} / \mathrm{mL}), 4(50 \mathrm{pg} / \mathrm{mL}), 5(100 \mathrm{pg} / \mathrm{mL}), 6(200 \mathrm{pg} / \mathrm{mL}), 7(400 \mathrm{pg} / \mathrm{mL})$ and serum sample by three batches reagents are presented in $(\mathbf{A}-\mathbf{G})$, respectively.

patients, 107 EBL patients, and 151 healthy subjects) by CLEIA. Our results demonstrated the potential of serum CST1 for the diagnosis of early ESCC patients, with 0.654 of AUC, and 31.25 of sensitivity $(92.64 \%$ specificity) higher than $16.07 \%-28.57 \%$ of sensitivity $(93.80$
95.35\% specificity) of three traditional tumor markers: CEA, CYFRA21-1 and SCC-Ag. Considering that the sensitivity of individual detection is relatively limited, we evaluated all possible combinations of serum CST1 and three traditional tumor markers: CEA, CYFRA21-1 

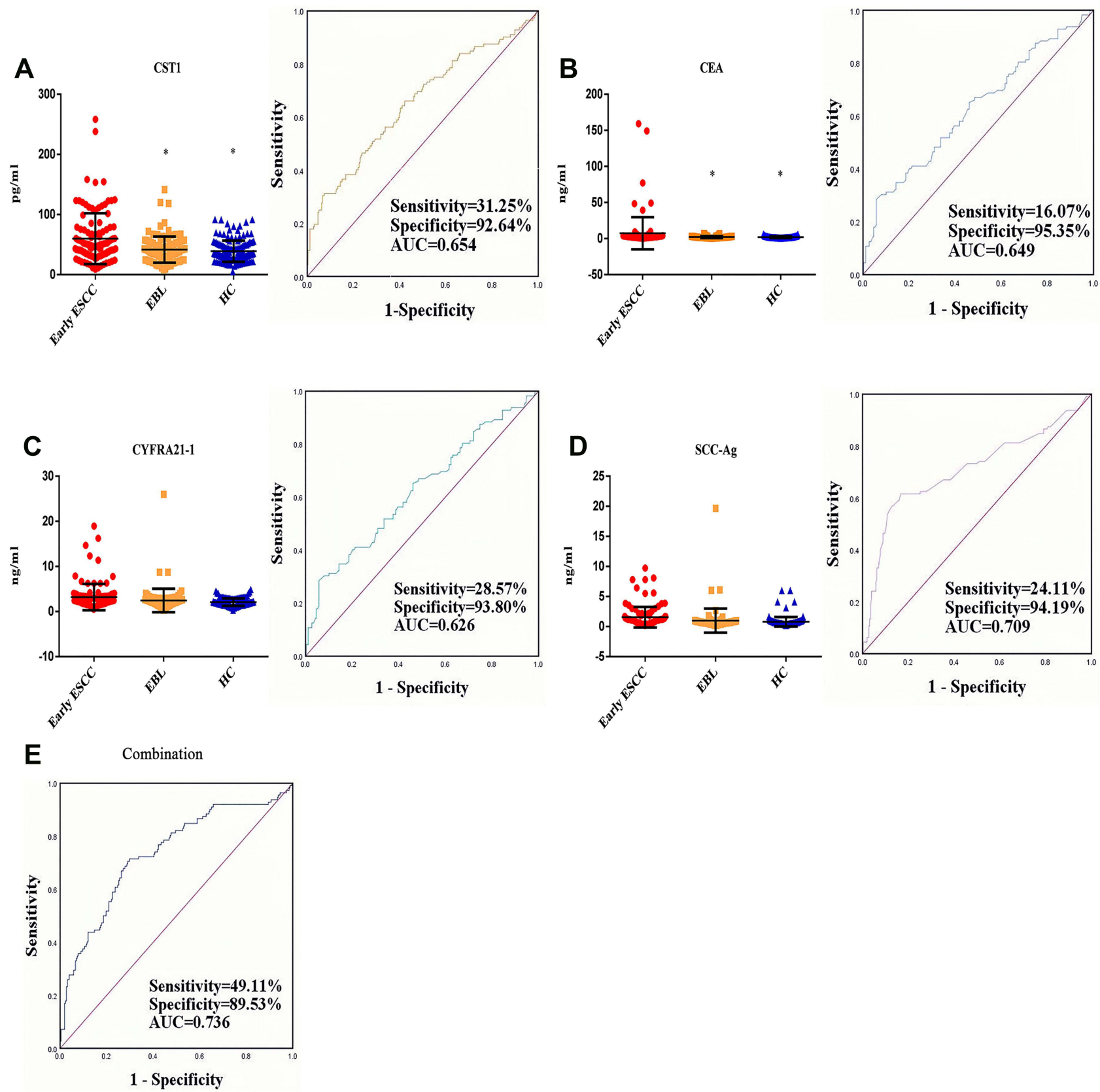

Figure 6 Diagnostic performances of serum CSTI and three traditional tumor markers CEA, CYFRA2I-I, and SCC-Ag for early ESCC patients. Boxplot, scatter and ROC curve for CSTI (A) CEA (B) CYFRA2I-I (C) and SCC-Ag (D). (E) ROC curve for an optimal panel of CSTI with CEA and SCC-Ag. *Compared with the early ESCC group, $P<0.05$.

and SCC-Ag. Encouragingly, the combination of CST1 with CEA and SCC-Ag was determined to be an optimal panel for the early diagnosis of ESCC, supported by its potential to improve AUC to 0.736, with up to $49.11 \%$ sensitivity at $89.53 \%$ specificity for early ESCC patients.
In conclusion, we successfully developed a CLEIA with good detection performance for serum CST1 and made clear the diagnostic value of serum CST1 for early ESCC patients. We then established an optimal panel of serum CST1 with CEA and SCC-Ag for further improvement of the early diagnostic performance for ESCC patients. 
Table 6 Comparison of Different Combinations of Serum CSTI and Three Traditional Tumor Markers for Early Diagnosis of ESCC Patients

\begin{tabular}{|l|l|c|c|c|c|}
\hline Index & AUC & SE & 95\% CI & Sensitivity (\%) & Specificity (\%) \\
\hline CEA, CYFRA2I-I & 0.669 & 0.032 & $0.586-0.712$ & 33.04 & 92.64 \\
CEA, SCC-Ag & $0.730^{\mathrm{a}}$ & 0.029 & $0.672-0.788$ & 29.46 & 92.25 \\
CEA, CSTI & 0.660 & 0.032 & $0.596-0.724$ & 37.50 & 91.09 \\
CYFRA2I-I, SCC-Ag & 0.705 & 0.031 & $0.644-0.766$ & 39.29 & 91.09 \\
CYFRA2I-I, CSTI & 0.659 & 0.032 & $0.596-0.722$ & 42.86 & 90.31 \\
SCC-Ag, CSTI & $0.730^{\mathrm{a}}$ & 0.030 & $0.673-0.788$ & 43.75 & 91.09 \\
CEA, CYFRA2I-I, SCC-Ag & 0.732 & 0.030 & $0.674-0.790$ & 40.18 & 89.53 \\
CEA, CYFRA2I-I, CSTI & 0.668 & 0.032 & $0.605-0.732$ & 44.64 & 88.76 \\
CEA, SCC-Ag, CSTI & $0.736^{\mathrm{b}}$ & 0.039 & $0.678-0.793$ & 49.11 & 89.53 \\
CYFRA2I-I, SCC-Ag, CSTI & 0.728 & 0.030 & $0.670-0.786$ & 48.21 & 89.15 \\
CEA, CYFRA2I-I, SCC-Ag, CSTI & 0.735 & 0.030 & $0.677-0.793$ & 50.89 & 87.98 \\
\hline
\end{tabular}

Notes: ${ }^{a}$ The highest AUC in the combined detection of any two indicators. ${ }^{\mathrm{b}}$ The highest AUC in the combined detection of any three indicators.

\section{Abbreviations}

ESCC, esophageal squamous cell carcinoma; EAC, esophageal adenocarcinoma; CST1, Cystatin SN; IHC, immunohistochemical; CLEIA, chemiluminescent enzyme immunoassay; CLIA, chemiluminescence immunoassay; EBL, esophageal benign lesions; $\mathrm{HC}$, healthy controls; $\mathrm{SD}$, standard deviation; RF, rheumatoid factor; $\mathrm{CV}$, coefficient of variation; TEa, total error allowance.

\section{Acknowledgments}

This work was supported by High-level Hospital Foster Grant of Fujian Provincial Hospital (Grant No. 2020HSJJ06), Medical Vertical Project of Fujian Province (Grant No. 2020CXB001).

\section{Disclosure}

The authors report no conflicts of interest in this work.

\section{References}

1. Wang C, Wang J, Chen Z, et al. Immunohistochemical prognostic markers of esophageal squamous cell carcinoma: a systematic review. Chin $J$ Cancer. 2017;36(1):65. doi:10.1186/s40880-017-0232-5

2. Arnold M, Soerjomataram I, Ferlay J, et al. Global incidence of oesophageal cancer by histological subtype in 2012. Gut. 2015;64 (3):381-387. doi:10.1136/gutjnl-2014-308124

3. Bray F, Ferlay J, Soerjomataram I, et al. Global cancer statistics 2018: GLOBOCAN estimates of incidence and mortality worldwide for 36 cancers in 185 countries. CA Cancer J Clin. 2018;68(6):394-424. doi: $10.3322 /$ caac. 21492

4. Pennathur A, Gibson MK, Jobe BA, et al. Oesophageal carcinoma. Lancet. 2013;381(9864):400-412. doi:10.1016/S0140-6736(12)60643-6

5. Domper Arnal MJ, Ferrández Arenas Á, Lanas Arbeloa Á. Esophageal cancer: risk factors, screening and endoscopic treatment in Western and Eastern countries. World J Gastroenterol. 2015;21(26):7933-7943. doi:10.3748/wjg.v21.i26.7933
6. Lagergren J, Ye W, Lagergren P, et al. The risk of esophageal adenocarcinoma after antireflux surgery. Gastroenterology. 2010;138 (4):1297-1301. doi:10.1053/j.gastro.2010.01.004

7. Kosugi S, Nishimaki T, Kanda T, et al. Clinical significance of serum carcinoembryonic antigen, carbohydrate antigen 19-9, and squamous cell carcinoma antigen levels in esophageal cancer patients. World J Surg. 2004;28(7):680-685. doi:10.1007/s00268-004-6865-y

8. Shimada H, Nabeya Y, Okazumi S, et al. Prognostic significance of CYFRA 21-1 in patients with esophageal squamous cell carcinoma. J Am Coll Surg. 2003;196(4):573-578. doi:10.1016/S1072-7515(02) 01905-1

9. Anastasi A, Brown MA, Kembhavi AA, et al. Cystatin, a protein inhibitor of cysteine proteinases. Improved purification from egg white, characterization, and detection in chicken serum. Biochem $J$. 1983;211(1):129-138. doi:10.1042/bj2110129

10. Abrahamson M, Barrett AJ, Salvesen G, et al. Isolation of six cysteine proteinase inhibitors from human urine. Their physicochemical and enzyme kinetic properties and concentrations in biological fluids. J Biol Chem. 1986;261(24):11282-11289. doi:10.1016/S00219258(18)67380-6

11. Barka T, Asbell PA, van der Noen H, et al. Cystatins in human tear fluid. Curr Eye Res. 1991;10(1):25-34. doi:10.3109/ 02713689109007608

12. Dickinson DP, Thiesse M, Dempsey LD, et al. Genomic cloning, physical mapping, and expression of human type 2 cystatin genes. Crit Rev Oral Biol Med. 1993;4(3-4):573-580. doi:10.1177/ 10454411930040034401

13. Feldman AS, Banyard J, Wu CL, et al. Cystatin B as a tissue and urinary biomarker of bladder cancer recurrence and disease progression. Clin Cancer Res. 2009;15(3):1024-1031. doi:10.1158/ 1078-0432.CCR-08-1143

14. Breznik B, Mitrović A, Lah T, et al. Cystatins in cancer progression: more than just cathepsin inhibitors. Biochimie. 2019;166:233-250.

15. Yoneda $\mathrm{K}$, Iida $\mathrm{H}$, Endo $\mathrm{H}$, et al. Identification of Cystatin $\mathrm{SN}$ as a novel tumor marker for colorectal cancer. Int J Oncol. 2009;35 (1):33-40.

16. Choi EH, Kim JT, Kim JH, et al. Upregulation of the cysteine protease inhibitor, cystatin $\mathrm{SN}$, contributes to cell proliferation and cathepsin inhibition in gastric cancer. Clin Chim Acta. 2009;406(12):45-51. doi:10.1016/j.cca.2009.05.008

17. Cui Y, Sun D, Song R, et al. Upregulation of cystatin SN promotes hepatocellular carcinoma progression and predicts a poor prognosis. J Cell Physiol. 2019;234(12):22623-22634. doi:10.1002/jcp.28828 
18. Jiang J, Liu HL, Liu ZH, et al. Identification of cystatin SN as a novel biomarker for pancreatic cancer. Tumour Biol. 2015;36 (5):3903-3910. doi:10.1007/s13277-014-3033-3

19. Dai DN, Li Y, Chen B, et al. Elevated expression of CST1 promotes breast cancer progression and predicts a poor prognosis. J Mol Med. 2017;95(8):873-886. doi:10.1007/ s00109-017-1537-1
20. Schwartz DR, Wu R, Kardia SL, et al. Novel candidate targets of beta-catenin/T-cell factor signaling identified by gene expression profiling of ovarian endometrioid adenocarcinomas. Cancer Res. 2003;63(11):2913-2922.

21. Chen S, Liu Y, Zhang K, et al. CST1 promoted gastric cancer migration and invasion through activating Wnt pathway. Cancer Manag Res. 2021;13:1901-1907. doi:10.2147/CMAR.S277770

\section{Publish your work in this journal}

Cancer Management and Research is an international, peer-reviewed open access journal focusing on cancer research and the optimal use of preventative and integrated treatment interventions to achieve improved outcomes, enhanced survival and quality of life for the cancer patient.
The manuscript management system is completely online and includes a very quick and fair peer-review system, which is all easy to use. Visit http://www.dovepress.com/testimonials.php to read real quotes from published authors. 